Le développement d'actions préventives contre la délinquance à Tokyo et à Osaka : une recomposition des liens de voisinage dans les territoires urbanisés du Japon?

東京、大阪における防犯活動の発展一都市部近隣ネットワークの再 構成についての検討

The Development of Crime Prevention Measures in Tokyo and Osaka: Restructuring Neighbourhood Bonds in Urban Areas of Japan

\title{
Naoko Tokumitsu
}

\section{OpenEdition}

\section{Journals}

Édition électronique

URL : http://journals.openedition.org/ebisu/2500

DOI : $10.4000 /$ ebisu. 2500

ISSN : 2189-1893

Éditeur :

Institut français de recherche sur le Japon (UMIFRE 19 MAEE-CNRS), Maison franco-japonaise

Édition imprimée

Date de publication : 15 décembre 2018

Pagination : 85-110

ISSN : 1340-3656

Référence électronique

Naoko Tokumitsu, «Le développement d'actions préventives contre la délinquance à Tokyo et à

Osaka : une recomposition des liens de voisinage dans les territoires urbanisés du Japon? », Ebisu [En ligne], 55 | 2018, mis en ligne le 15 décembre 2018, consulté le 24 avril 2019. URL : http:// journals.openedition.org/ebisu/2500 ; DOI : 10.4000/ebisu.2500 


\section{Le développement d'actions préventives contre la délinquance à Tokyo et à Osaka}

Une recomposition des liens de voisinage dans les territoires urbanisés du Japon?

Naoko TокUмітsu

東京、大阪における防犯活動の発展

都市部近隣ネットワークの再構成についての検討

徳光直子

The Development of Crime Prevention Measures in Tokyo and Osaka Restructuring Neighbourhood Bonds in Urban Areas of Japan

Naoko Toкumitsu

Mots-clés : Chōnaikai, police, prévention, insécurité, fabrique de la ville, liens de voisinage.

L'auteure : Naoko Tokumitsu est docteure de l'EHESS en sociologie et maître de langue à l'université Paris Diderot (UFR LCAO). Ses recherches portent sur le processus par lequel la police japonaise a incité les habitants à participer au maintien de la sécurité locale, dans un contexte d'urbanisation.

Résumé : Malgré une montée de l'urbanisation ayant pour conséquence de défaire des liens préexistants tels que ceux des communautés de quartier appelées chōnaikai, nous pouvons aujourd'hui observer un développement du maillage des liens de voisinage dans les grandes villes avec pour finalité affichée la prévention de l'insécurité. Reposant sur l'analyse des archives depuis les années 1970 et sur des enquêtes de terrain réalisées à Tokyo, Osaka et à Kōbe, cet article s'attache à retracer la manière dont ce phénomène $\mathrm{a}$ été encouragé en interaction avec la police et les urbanistes ainsi qu'avec le gouvernement. S'y trouve également exposé le processus suivant lequel les habitants inscrivent leurs actions préventives au sein du quartier, avec une description des différences qui peuvent exister selon leur statut et les liens qu'ils visent à tisser au sein du quartier. 
】 キーワード

町内会、警察、予防、犯罪不安、まちづくり、 近隣ネットワーク

\section{著者}

フランス社会科学高等研究院にて社会学博士 号を取得。パリ・ディドロ大学講師。都市化 の進展の中で警察が地域住民の協力を得てき た過程を研究している。
要旨

都市化に伴い、町内会等の既存の社会的紐帯 の衰退が指摘されている。しかし一方で、犯罪 不安を背景に防犯活動による近隣ネットワーク の発展が大都市で見受けられる。本稿では 70 年代以降の資料分析、および東京、大阪、神 戸でのフィールドワークをもとに、警察、都市 計画家、政府がいかに連関し、この現象を推 し進めてきたかを考察する。また、地域住民が 防犯活動をどのように社会生活の中に組み入れ たか、地域における地位と紐帯の相違性に注目 して分析する。
\ Keywords: Chōnaikai, police, prevention, public insecurity, community building, neighbourhood bonds.

The Author: Naoko Tokumitsu holds a $\mathrm{PhD}$ in sociology from EHESS and maître de langue at Paris Diderot University (UFR LCAO). Her studies focus on the process by which the Japanese police have encouraged residents to participate in local security measures in the context of urbanization.

Abstract: Despite rising urbanization and its tendency to undo pre-existing social bonds in neighbourhood commu- nities like the chonaikai, local bonds are being developed in big cities as a means of preventing public insecurity. Based on the analysis of archives from the 1970 s onwards and on fieldwork carried out in Tokyo, Osaka and Kōbe, this article studies the way this phenomenon has been supported by the police, urban planners and the government. In addition to explaining how the preventive measures adopted by residents take place within the neighbourhood, it describes the differences that may exist depending on the residents' status and the neighbourhood bonds they aim to develop. 


\title{
Le développement d'actions préventives contre la délinquance à Tokyo et à Osaka Une recomposition des liens de voisinage dans les territoires urbanisés du Japon?
}

\author{
Naoko TOKUMITSU*
}

\section{Introduction}

Le Japon connaît aujourd'hui une baisse du taux d'adhésion aux organisations locales dites traditionnelles telles que les communautés de quartier appelées chōnaikai (町内会) ou jichikai (自治会) ${ }^{1}$. En effet, malgré la décision du Commandement suprême des forces alliées (GHQ) de les dissoudre en 1947, la majorité des chōnaikai ont continué à exister de manière

1. En s'appuyant sur une comparaison internationale, Nakata (2000) remarque qu'au Japon, les communautés de quartier se distinguent par une moindre importance du volontariat, pour deux raisons : premièrement, toutes les personnes résidant ou faisant commerce de biens ou de services au sein du périmètre établi sont invitées à être membres des chōnaikai, même si l'adhésion n'est pas, en soi, obligatoire. Ainsi, le taux d'adhésion est plus important lorsque les liens de voisinage sont fortement présents; deuxièmement, ils entretiennent un lien étroit avec les collectivités locales et remplissent différentes fonctions (faire passer des annonces municipales d'un ménage à l'autre, collecter des dons, participer à la gestion de l'éclairage public, organiser le dépôt des déchets, veiller à la propreté du quartier, en assurer la sécurité, organiser les événements propres au quartier, etc.). Après la période d'occupation américaine, de nombreux chōnaikai ont repris leurs activités et certains se sont renommés jichikai (自治会) afin de s'en démarquer (Ajisaka 2006).

* Maitre de langue à l'université Paris Diderot (UFR LCAO). 
informelle, parfois en changeant de nom, afin de gérer certains aspects du quotidien tels que la sécurité, l'hygiène et les événements de sociabilité, ainsi que pour entretenir un lien avec l'administration² (Iwasaki et al. 1989). Leur nombre a par ailleurs augmenté pendant la période de Haute Croissance économique, en particulier dans les zones de périurbanisation. Jusqu'au milieu des années 1990, plus de $60 \%$ de la population adhérait aux chōnaikai (fig. 1), ce qui n'est plus le cas aujourd'hui, surtout en zone urbaine. Ainsi, d'après une enquête nationale réalisée par Tsujinaka et al. (2009), entre 2006 et 2007, parmi les chōnaikai qui enregistraient moins de $50 \%$ d'adhésion, 72,6\% se trouvent dans les zones urbaines où se concentrent notamment l'habitat collectif et les commerces, avec une augmentation de la population par rapport aux zones rurales ${ }^{3}$. Ils se caractérisent par une part importante de célibataires actifs, ainsi que par un faible taux de capital social, avec moins d'échanges entre voisins et une faible participation aux activités locales.

2. En s'appuyant sur les enquêtes réalisées en 1952 par l'ancien office du Cabinet (sōrifu 総理府), Iwasaki et al. (1989) montrent que depuis la dissolution des chönaikai jusqu'à leur rétablissement, $1,2 \%$ ont continué à exister, $77,9 \%$ se sont rétablis en moins de trois mois, 3,8 \% ont été rétablis avant 1947, 6,3 \% avant 1948, 2,9\% avant $1949,2,9 \%$ avant $1950,1,9 \%$ avant $1951,1,9 \%$ n'ont pas répondu et 1,2 \% ne se sont pas rétablis.

3. Il s'agit de chōnaikai de type "ancien et urbain" et de type "nouveau et urbain", selon la typologie de Tsujinaka et al. (2009), qui distingue quatre types de chōnaikai, à partir d'une enquête nationale : 1) le type ancien et rural : la taille de chaque chōnaikai est petite; 2) le type nouveau et non-urbain de taille relativement petite, né après le milieu des années 1990 notamment dans les petites villes; 3) le type ancien et urbain de grande taille (62,1\% de ces chönaikai comptent plus de deux cents foyers); 4) le type nouveau et urbain de grande taille (58,3\% comptent plus de deux cents foyers). 


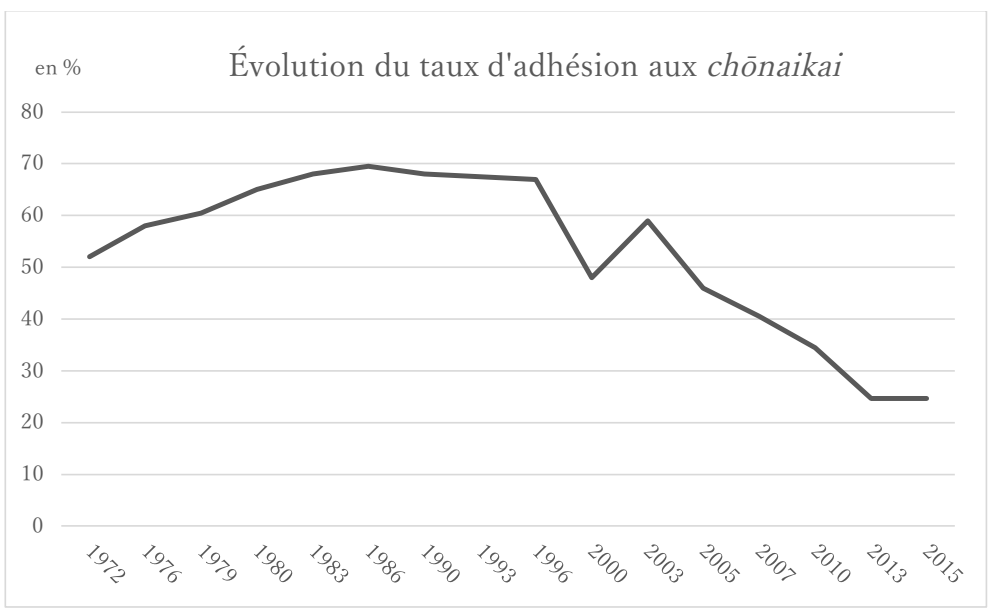

Fig. 1

Évolution du taux d'adhésion aux chōnaikai.

Source : Tsujinaka et al. (2009). Complété par l'auteure pour les données entre 2010 et 2015, à partir de la source : Enquêtes nationales réalisées par l'association pour la promotion des élections transparentes (Akarui senkyo suishin kyōkai 明るい選挙推進協会)

Cette enquête montre aussi que ces chönaikai accomplissent davantage d'activités pour la prévention de la délinquance et nouent un partenariat plus dense que la moyenne avec les associations de prévention (bōhan kyōkai 防犯協会), la police et les pompiers. Ce phénomène s'observe à travers le développement de nouveaux groupes de voisinage dans les grandes villes ayant pour finalité affichée la prévention de l'insécuritét. D'après les statistiques établies par l'Agence nationale de la police japonaise, la proportion des participants a augmenté dans le sillage de la mise en œuvre de ce plan : en 2003, on recensait environ 180000 participants, tandis qu'en 2016, leur

4. Les villes qui comptent plus de 100000 participants aux actions préventives correspondent aux départements suivants : Tokyo, Saitama, Chiba, Kanagawa, Aichi, Osaka, Hyōgo et Fukuoka. Cette politique policière s'inscrit également dans un contexte de crainte d'un manque d'effectifs : dans le livre blanc de l'Agence nationale de la police en 2005, celle-ci précisait qu'il y avait un policier pour cinq cent vingt personnes, contrairement à des pays tels que les États-Unis (un pour trois-cent-cinquante-trois en 2000) ou le Royaume-Uni (un pour trois-cent-trente-sept en 2002). 
nombre atteignait près de 2730000 personnes 5 . Leur profil se caractérise par une tranche d'âge plutôt élevée : 51,2\% ont entre 60 et 69 ans, 18,1\% entre 50 et 59 ans, et enfin 14,1\% entre 70 et 79 ans.

La diffusion nationale de ces actions s'inscrit dans un plan du gouvernement mis en œuvre à partir de 2003, parallèlement à la présentation du "programme pour des mesures de sécurité urgentes" (kinkyū chian taisaku puroguramu 緊急治安対策プログラム) par l'Agence nationale de la police, qui faisait le constat d'une montée de la criminalité et du sentiment d'insécurité ainsi que d'une baisse de la confiance envers la police ${ }^{6}$. Ce plan visait à renforcer la coopération entre les habitants, la police et les collectivités locales, mais aussi avec le secteur de la sécurité privée. Il prônait le renforcement des liens de solidarité au sein des quartiers en encourageant les actions préventives bénévoles. Or, si la plupart de ces actions avaient pour objectif principal de réaliser des patrouilles de groupe au sein du quartier, elles s'accompagnent souvent de mesures d'hygiène et d'une dimension d'éducation morale : pratiques de nettoyage de rue, salutations visant à développer la sociabilité des individus, notamment des enfants, etc. ${ }^{7}$. Leur champ d'action s'étend également à l'organisation des événements culturels du quartier, dans le but d'animer les échanges interpersonnels, ce qui s'inscrit dans l'idée de fabrique de la ville, avancée par les politiques publiques locales. Sur le terrain, cet objectif de sociabilité entre voisins peut parfois même être présenté comme la finalité principale des actions. Encouragées par des initiatives politiques successives, ces actions peuvent certes avoir eu

5. Le recensement repose sur la prise en compte des groupes de plus de cinq personnes, réalisant au moins une activité notable par mois, en dehors des réunions.

6. Le nombre de délits enregistrés a atteint un pic en 2002 en matière de délinquance de rue (vandalisme, vols...), même si ces données sont à prendre avec précaution (voir note 8). La police a d'abord craint la montée du sentiment d'insécurité, notamment apparue en décembre 1995 dans un sondage de l'office du Cabinet (naikakufu 内閣府). La baisse de confiance est liée à une dégradation de l'image de la police entre 1999 et 2002, notamment après le meurtre d'une étudiante par un «stalker» (harceleur) dans la ville d'Okegawa, à la suite duquel certains médias avaient critiqué l'attitude de la police face à la victime et à sa famille. Nous pouvons aussi relever la hausse subite du nombre d'articles de journaux contenant les mots "police» et «scandale» (fushōji 不祥事), à partir de 1999 (Hamai 2006).

7. En $2016,78,8 \%$ des groupes réalisent des patrouilles à pied et 75,2 \% veillent sur les enfants sur le chemin de l'école. 
un effet positif sur le taux de délinquance de rue, mais, initialement, ce taux n'avait pas tendance à augmenter, comme le montre l'Agence nationale de la police (Hamai 2013). Il existe ainsi un décalage entre des discours alarmants portant sur les menaces à l'ordre public et une réalité statistique de la criminalité qui s'avère en baisse ${ }^{8}$.

Dans la mesure où peu de recherches décrivent la manière dont ces actions ont été mises en œuvre sur le terrain, en interaction avec les autres organisations, il nous a semblé pertinent d'examiner dans cet article la manière dont les acteurs publics ont fait appel aux habitants et la façon dont ces derniers se sont mobilisés sans que cela leur soit imposé ${ }^{\text {. Nous }}$ nous sommes appuyés à cette fin sur l'analyse de documents de source administrative, tels que les «livres blancs» publiés par l'Agence nationale de la police depuis 1973 jusqu'à la mise en place d'un soutien aux activités bénévoles de prévention en 2005 . Viennent en complément des enquêtes de terrain menées auprès de quinze groupes, sélectionnés à partir de l'analyse des archives de trois cent cinquante-deux groupes, l'enjeu étant d'étudier la manière dont les directives annoncées ont été mises en œuvre à travers les actions bénévoles des habitants.

8. Hamai (2013) estime que la baisse du taux de délinquance de rue est en partie liée aux actions préventives, mais que cela est difficile à évaluer car les chiffres sont publiés par la police qui, notamment depuis 2002, cherche à réduire le nombre de crimes et de délits enregistrés. Il montre que, contrairement à la montée alléguée de la criminalité, les enquêtes de victimation indiquent que celle-ci est en baisse de 1995 à 2008, excepté pour les vols de voitures. Kawai (2006) montre quant à lui que cette augmentation est d'abord liée à un biais statistique : suite au meurtre d'une étudiante par un harceleur d'Okegawa, la police aurait commencé à accepter davantage les plaintes, ce qui aurait augmenté le nombre de crimes enregistrés.

9. Par exemple, Shimizu (2007) détaille l'évolution des politiques policières depuis 1993. Il fait notamment la lumière sur l'histoire de la prévention situationnelle. Il convient également d'évoquer les travaux d'Obinata (1993) décrivant la manière dont la police s'est rapprochée des habitants au début du $\mathrm{Xx}^{\mathrm{e}}$ siècle et la façon dont les groupes de vigilance (jikeidan 自警団) se sont formés. 


\section{La participation progressive des habitants à la prévention de l'insécurité}

\section{La genèse des mesures policières visant à encourager la solidarité de voisinage}

Afin de comprendre le développement des groupes de prévention, nous rappellerons brièvement le contexte historique du développement des actions préventives policières au sein de l'espace public japonais. À l'époque d'Edo, les quartiers urbains étaient organisées en communautés de voisins, désignées par le caractère chinois $c h \bar{o}$ (町) ${ }^{10}$, qui se chargeaient du maintien de l'ordre et de l'entretien de diverses installations (Carré 2003). Dans ce cadre, le système des groupes de cinq (gonin gumi 五人組) s'est généralisé dans l'ensemble du pays : ceux-ci visaient à ce que les voisins se surveillent et s'entraident et à ce qu'ils prennent une responsabilité commune en cas d'écart par rapport aux règles établies ${ }^{11}$. Ce système a cependant perdu son rôle institutionnel après la Restauration de Meiji mais a continué à exister de manière informelle, en tant que groupe d'entraide entre voisins. Vers la fin du XIX ${ }^{\mathrm{e}}$ siècle, les communautés de quartier ont commencé à prendre part au maintien de l'ordre : d'une part afin que les mœurs ${ }^{12}$ ne soient pas perçues comme sauvages pour le regard occidental dans un contexte d'ouverture du pays; d'autre part, pour empêcher l'expansion d'une épidémie de choléra, les syndicats d'hygiène (eisei kumiai 衛生組合), formes d'origine

10. Carré (2003) remarque que les dimensions d'un chō étaient très variables et que le même caractère pouvait également désigner la totalité des quartiers bourgeois d'une ville seigneuriale, voire un centre urbain secondaire dans son ensemble.

11. Cette surveillance avait pour objectif d'interdire le christianisme, de contrôler plus sévèrement les guerriers sans attache seigneuriale, ainsi que d'améliorer la collecte des impôts. Avec la stabilisation du régime shogunal, s'est progressivement adjointe une dimension morale et économique concernant par exemple la gestion d'un budget, l'apprentissage de l'assiduité dans le travail, et une éducation morale mettant l'accent sur la justesse, l'honnêteté et la bienfaisance (Yoshihara 2011).

12. Cela comprenait le commerce ambulant, la prostitution, les spectacles, le manque d'hygiène, les prêteurs sur gages (shichiya 質屋), les comportements jugés déviants, etc. (Obinata 1993) 
des chōnaikai, ayant été formés en vue de la surveillance mutuelle ${ }^{13}$. Plus tard, en 1940, dans un contexte de mobilisation nationale pour la Seconde Guerre mondiale, le ministère des Affaires intérieures a placé les chōnaikai à la base de la hiérarchie administrative. Mais ces derniers chapeautaient eux-mêmes les associations de voisinage (tonari gumi 隣組) composées d'environ dix foyers, chargées par exemple de contrôler la circulation des idées subversives, de distribuer des rations de nourriture, de veiller sur la sécurité publique et l'hygiène, de prévenir les incendies, etc. Ces communautés de voisinage (comprenant donc les chōnaikai et les tonari gumi) ont été ensuite dissoutes par le GHQ en 1947. Mais elles ont bien continué à exister de manière informelle, notamment en matière de prévention de la délinquance et des incendies, tout en perdant le caractère obligatoire d'adhésion à ces organisations. Les chōnaikai se sont également distingués des associations de prévention de la délinquance (bōhan kyōkai 防犯協会) instaurées dans chaque îlot policier. Critiquant l'instrumentalisation de ces associations, ils cherchaient à garantir leur autonomie face aux pouvoirs publics (Hishiyama 2014) : il était ainsi rare d'observer, dans les années 1950 et 1960, des associations sécuritaires regroupant des habitants sur le modèle d'aujourd'hui.

Dans son livre blanc de 1976, la police craignait une transformation et une détérioration du tissu social urbain, estimant qu'elles étaient de nature à "compliquer la collecte des informations par ses services ${ }^{14}$ ». Elle ne manquait pas de dénoncer à cette occasion la montée « de l'individualisme, d'un manque de reconnaissance mutuelle et de solidarité, ainsi [qu'un] anonymat grandissant ${ }^{15}$ ". Elle pointe, dans ce contexte, l'influence du phénomène d'urbanisation et le développement des technologies de l'information et de la communication ainsi que l'internationalisation de la société, comme ayant "amené la destruction des communautés locales, lesquelles avaient une force dissuasive ${ }^{16}{ }^{\prime}$. Nous observons ainsi à cette époque, de la part de

13. Les syndicats d'hygiène se sont développés au niveau national notablement après mars 1897, année d'adoption de la loi pour la prévention de l'épidémie.

14. Keisatsu hakusho 警察白書 (Livre blanc de l'Agence nationale de la police), 1976.

15. Ibid.

16. Keisatsu hakusho 警察白書 (Livre blanc de l'Agence nationale de la police), 1979. 
la police, une plus grande volonté de participer aux différentes activités de quartier comme celles proposées par les chönaikai.

Cette participation policière a pour contexte un changement d'orientation politique dans les années 1970. En effet, dans les années 1950-1960, l'étalement urbain de la criminalité remettait en question les modalités d'organisation du dispositif policier. Une modernisation sur le modèle américain a alors été mise en place avec la diminution des bureaux de police (hashutsujo 派出所 ou chüzaisho 駐在所) et l'augmentation du nombre de voitures de patrouille. Cependant, à partir des années 1970, la revalorisation de ce système survenue en Europe et aux États-Unis a amené la police à vouloir remettre en avant les bureaux de police ainsi que leur fonction de prévention "à la japonaise» (Watanabe 2005).

Dans les années 1980, la police fait le lien entre l'aggravation du taux de délinquance juvénile, l'augmentation ou la baisse des familles nucléaires et le changement de valeurs qui aurait accompagné le développement économique. Craignant le développement d'une mentalité influencée par l'Occident, elle a alors associé ce phénomène à une tendance des adolescents à rechercher des plaisirs "éphémères» (setsunateki せつな的), manifestant un manque de «maîtrise de soi» (kokkishin 克己心) ${ }^{17}$. La police dénonçait la transformation des communautés locales et de la structure familiale qui aurait contribué à "diminuer les fonctions d'autogestion des problèmes, d'entraide et de dissuasion de la délinquance ${ }^{18}$ ". La police souligne l'importance des efforts que les habitants doivent fournir pour l'aider à résoudre des problèmes tels que la délinquance juvénile ou les nuisances sonores ${ }^{19}$.

En raison des craintes liées au vieillissement de la population, cette consigne a été mise en avant dans le cadre des politiques à destination des personnes âgées, invitées à devenir les acteurs des actions préventives. En 1986, le gouvernement a publié un «Bilan des mesures générales pour une société vieillissante ${ }^{20}$ » visant à garantir l'emploi et l'insertion sociale des personnes âgées (par la formation, les loisirs, etc.), ainsi que les conditions

17. Keisatsu hakusho 警察白書 (Livre blanc de l'Agence nationale de la police), 1981.

18. Keisatsu hakusho 警察白書 (Livre blanc de l'Agence nationale de la police), 1984.

19. Ibid.

20. Chöju shakai taisaku taikō 長寿社会対策大綱 (Bilan des politiques publiques face à une société vieillissante), 6 juin 1986. 
d'une vie «sûre et agréable» reposant sur l'autonomie et la solidarité au sein de leurs communautés. En prenant appui sur ces orientations politiques, la police a envisagé de protéger davantage ces personnes au moyen de dispositifs variés : communication d'informations relatives aux divers types de délits, diffusion de conseils utiles pour se prémunir à la fois de la délinquance et des accidents de la route, renforcement de la participation à la vie du quartier, etc. ${ }^{21}$. Ce programme traduit l'idée que la participation des bénévoles à ces activités peut exercer des effets bénéfiques sur leur propre raison d'être et, in fine, sur leur bien-être quotidien. Plus spécifiquement, il s'agit de montrer que «les échanges interpersonnels peuvent faire advenir une société participative dans laquelle solidarité du quartier et dynamisme social se renforcent ${ }^{22}$ ». Les personnes âgées sont donc considérées ici tout à la fois comme les «bénéficiaires» de cette protection et comme des «acteurs» capables de revitaliser l'esprit de solidarité d'un quartier.

Il faut dire enfin que la fin des années 1980 se caractérise par l'émergence d'une forte crainte à l'égard de la mondialisation. Des facteurs culturalistes sont avancés pour expliquer la notion d' "exception japonaise", qu'expriment notamment un taux de criminalité en moyenne plus faible que dans les autres pays de l'OCDE, «le respect de la loi, la croissance économique, [...] une éducation de qualité, la présence d'un contrôle informel de quartier, [...] et enfin l'action favorable des services de police et de la justice. Toutefois, toutes ces conditions ne sont pas pérennes ${ }^{23}$ ". Comme on peut le constater, les arguments présentés par la police sont d'ordres culturel, pédagogique, conjoncturel, institutionnel.

À partir des années 1990, aux inquiétudes liées au vieillissement de population s'ajoute la méfiance vis-à-vis des résidents étrangers qui auraient "affaibli les fonctions d'entraide de la communauté locale ${ }^{24}$ » et des organisations criminelles étrangères s'implantant au Japon. Cela conduit la police à affirmer, à plusieurs reprises, que n'importe qui peut, à tout moment, être victime d'un crime. Cela peut se lire par exemple à travers l'emploi récurrent

21. Keisatsu jichō imei tsūtatsu 警察次長依命通達 (Avis officiel du sous-directeur de l'Agence nationale de la police), 21 juillet 1986.

22. Chöju shakai taisaku taikō 長寿社会対策大綱 (Bilan des politiques publiques face à une société vieillissante), 6 juin 1986.

23. Hanzai hakusho 犯罪白書 (Livre blanc sur le crime), 1989.

24. Keisatsu hakusho 警察白書 (Livre blanc de l'Agence nationale de la police), 1992. 
du terme anshin 安心 (paisible). Le projet «Cinq ans pour construire un pays ayant une bonne qualité de vie - à la recherche d'une cohabitation avec la société mondiale ${ }^{25}$ ", établi en juin 1992, se rattache plus particulièrement à cet aspect de la question, et, outre le confort matériel, souligne l'importance de la «richesse morale» (seishinteki na yutakasa 精神的な 豊かさ), décrite comme ayant été négligée pendant la période de croissance économique. Cette richesse morale se développe par la participation aux événements sportifs et culturels, par l'attention portée aux autres, présentée comme nécessaire à l'épanouissement de la société, etc. La volonté de faire avancer la politique du "point de vue des habitants du quartier ${ }^{26}$ " justifie la participation active de ceux-ci.

\section{2. Élargissement de la définition de la prévention et renforcement des aspects moraux de la sécurité}

À partir de 1994, on a demandé au niveau local que des efforts soient fournis pour inclure dans l'action de ces communautés de quartiers la prévention de tout risque pesant sur les habitants tels que les accidents et les catastrophes naturelles. De façon significative, la police a également institué à cette époque un département de sécurité de la vie quotidienne (seikatsu anzenbu 生活安全部). Dans ce cadre, elle a vivement encouragé les partenariats entre habitants, collectivités locales et policiers, afin de renforcer le volet préventif contre différents types de délits tels que les cambriolages, la délinquance juvénile et le démarchage illégal.

Un grand nombre de ces partenariats a effectivement vu le jour suite au séisme de Hanshin-Awaji en 199527. Des organisations locales telles que les chōnaikai ou d'autres bénévoles ont effectué des patrouilles en collaboration

25. Projet seikatsu taikoku gokanen keikaku - chikyū shakai to no kyōzon o mezashite 生活大国5か年計画 - 地球社会との共存を目指して, approuvé en Conseil des ministres le 30 avril 1992.

26. Keisatsu hakusho 警察白書 (Livre blanc de l'Agence nationale de la police), 1994.

27. Lors du tremblement de terre de Hanshin-Awaji, communément appelé "séisme de Kōbe», de nombreux habitants, coupés de toute infrastructure, ont été obligés de s'entraider pendant une longue période dans des lieux de refuge sommaires, ce qui les a conduits à s'organiser entre eux. 
avec les policiers dans les zones sinistrées, en vue d'assurer la sécurité des biens et d'essayer de répondre aux besoins (nourriture, soins, isolement, etc.) des victimes. La fonction de soutien psychologique de ces patrouilles a été positivement soulignée par la police. En outre, la nécessité d'adopter de nouvelles mesures de lutte contre l'insécurité a été réaffirmée après l'attentat au gaz sarin survenu dans le métro de Tokyo en $1995^{28}$, puis, la même année, suite à la tentative d'assassinat par arme à feu du Commissaire général de l'Agence nationale de la police ${ }^{29}$.

L'expérience de ce séisme a également exercé une influence déterminante sur la prévention de la délinquance en contexte urbain. À partir de 1997, plusieurs réunions consultatives ${ }^{30}$ portant sur la «fabrique d'une ville sûre et paisible» se sont tenues entre spécialistes des catastrophes naturelles, de criminologie, de droit, des politiques de logement et de l'urbanisme. Elles ont choisi pour cela les modèles nord-américain et britannique ${ }^{31}$, en invoquant notamment la théorie de la "vitre cassée ${ }^{32}$ ", décalquée sur le modèle d'origine visant à réduire des facteurs d'ordre environnemental, qui, sans être criminogène, a été plutôt transposée en termes moraux. Dans ce cadre, le rôle des salutations a également été jugé essentiel pour renforcer l'attention mutuelle entre voisins, la prévention de la délinquance, l'entraide en

28. Suite à cet attentat, une affiche appelant à la vigilance du public a été diffusée largement par l'Union nationale des associations de prévention (Zenkoku bōhan kyōkai rengōkai 全国防犯協会連合会).

29. Cette tentative d'assassinat a d'une part alimenté la peur d'un développement des armes à feu lié à l'internationalisation; d'autre part, elle a été perçue par l'Agence nationale de la police comme une provocation à son égard, signifiant que son autorité était menacée.

30. Ces réunions consultatives ont été organisées par l'Agence nationale de la police et le ministère de la Construction (actuellement le ministère du Territoire, des Infrastructures, des Transports et du Tourisme).

31. Il s'agit également, dans le cas britannique du NHBC (National House Building Council), du projet "Secured by Design», d'activités d'agents tels que le "crime prevention design advisor" ou encore de l' "architectural liaison officer".

32. Cette théorie, appelée "broken windows» en anglais, fut élaborée en 1982 par les criminologues américains J. Q. Wilson et G. Kelling. Elle indique que "dans le cas où une vitre brisée n'est pas remplacée, toutes les autres vitres connaissent bientôt le même sort». L'idée principale est que, dès que se multiplient les signes d'abandon, des actes de vandalisme surviennent, eux-mêmes suivis de comportements tels que des vols ou des agressions (Roché 2000). 
cas de catastrophes naturelles. Ont alors été valorisés les organisateurs des événements tels que les fêtes locales, ou les responsables de l'entretien des espaces verts, signalant ainsi aux visiteurs la présence d'habitants attentifs à leur espace de vie en communauté. Le rôle crucial de la présence humaine et du bon voisinage de quartier a été régulièrement rappelé, tel un leitmotiv, au cours des différentes réunions de la commission concernée, arguant du fait que «l'auto-défense (jiko bōei 自己防衛) peut isoler les individus et augmenter le sentiment d'insécurité33».

La série de meurtres commis par le jeune Sakakibara Seito 酒鬼蓄薇聖斗 a renforcé la moralisation de la politique de sécurité, tout en soulignant le rôle joué par les chōnaikai is. Le discours relayé par les médias fait généralement de Sakakibara la victime d'un système scolaire inhumain et générateur de malaise (rythme d'apprentissage pour les concours, obligation d'adopter une bonne conduite pour obtenir de meilleures évaluations scolaires, etc.) (Serizawa 2006). Ceci a conduit la police à dénoncer les quatre problèmes sociaux pouvant conduire à la délinquance : 1 ) environnement social considéré comme nocif (développement de services incitant les jeunes à sortir tard le soir, diffusion de contenus pornographiques ou violents, etc.) ; 2) affaiblissement des normes de santé (normalisation de l'alcool, du tabac, des drogues, etc.) ; 3) appauvrissement des échanges entre parents et enfants ainsi qu'entre enseignants et élèves; 4) relâchement des liens au sein de la communauté locale (chiiki shakai 地域社会 ${ }^{35}$ ). Or, à la suite des crimes de Sakakibara Seito, le chōnaikai du quartier Kitasuma, concerné par les meurtres, a commencé à mettre en place des actions préventives, en animant des événements de quartier, en montant des patrouilles, en assurant la

33. Anzen anshin machizukuri jissen shuhō chōsa kentō iinkai, dai 3 kai iinkai 安全· 安心まちづくり実践手法調査検討委員会、第3回委員会 (Résumé de la troisième réunion de la commission de recherches et de réflexion sur les méthodes pratiques de fabrique de la ville sûre et sans source d'inquiétude), février 1999.

34. Il s'agit de plusieurs meurtres commis par un jeune collégien de 14 ans (qui s'est donné le surnom de Sakakibara Seito 酒鬼著薇聖斗), ayant tué deux écoliers et blessé trois autres. Cette affaire a attiré l'attention de l'opinion publique car l'auteur était décrit comme un collégien "comme les autres" (futsū no ko 普通の子). Ses actes marqués par une grande cruauté ont pu conduire une partie de l'opinion à mettre en cause la pertinence d'une justice pénale protectrice des mineurs.

35. Keisatsu hakusho 警察白書 (Livre blanc de l'Agence nationale de la police), 2001. 
surveillance des enfants à la sortie de l'école, etc. ${ }^{36}$. Ses actions ont été alors présentées par les médias comme un modèle, comme l'une des réponses appropriées à l’augmentation de la criminalité.

\section{La place centrale de l'insécurité dans les débats politiques et le rôle des collectivités locales}

C'est en 2003 que l'État s'est véritablement saisi de cette notion de «fabrique d'une ville sûre et paisible». Une réunion interministérielle de lutte contre la criminalité ( hanzai taisaku kakuryō kaigi 犯罪対策閣僚会議) a eu lieu en décembre, autour du mot d'ordre suivant: «Pour que le Japon redevienne le pays le plus sûr du monde» ("Sekai ichi anzen na kuni, Nippon» no fukkatsu o mezashite「世界一安全な国、日本」の復活を目指して). Citant Guillaume de Humboldt, le gouvernement a alors avancé l’idée que «la liberté n'exist[ait] pas sans sécuritée " et a assuré que le fait de garantir la sécurité était crucial si l'on souhaitait encourager les activités économiques et le développement social. Suite à cette réunion, deux premiers axes de prévention ont été déterminés : le premier axe concerne le soutien aux actions des habitants visant à assurer la sécurité en favorisant la diffusion des informations, mais aussi en attribuant des subventions spéciales afin que les habitants puissent acquérir de l'équipement. Le deuxième axe concerne l'aménagement d'un environnement social empêchant la criminalité. L'État demande alors non seulement un renforcement de la présence policière dans la rue mais aussi une coopération active des habitants, qui sont invités à ne pas ignorer le moindre délit. Ce plan d'action est suivi, en juin 2005, d'un projet nommé «Fabrique d'une ville sûre et paisible : projet de développement au niveau national» (anzen anshin machizukuri zenkoku tenkai puran 安全·安心なまち づくり 全国展開プラン). Est alors décidée la création d'un «modèle» de réalisations pionnières afin de développer des actions similaires dans d'autres

36. Enquête de terrain réalisée en 2016 dans le quartier de Kitasuma auprès du président du chōnaikai.

37. "Plan d'action pour bâtir une société forte contre la criminalité (hanzai ni tsuyoi shakai no jitsugen no tame no kōdo keikaku 犯罪に強い社会の実現のための行動計画)", décembre 2003. 
villes. Concrètement, des subventions prioritairement attribuées à certaines activités sélectionnées pour leur dynamisme et un prix du Premier ministre ont été institués, avec l'objectif de récompenser les individus ou les groupes ayant contribué à la promotion de ce machizukuri3i. L'État essaie donc d'impliquer davantage les collectivités locales, tout en manifestant sa reconnaissance à l'égard d'individus ayant contribué de manière significative au maintien de la sécurité.

Enfin, il faut de nouveau mentionner le rôle déterminant joué par l'actualité et les faits divers. Ainsi, suite à une série d'assassinats d'enfants ayant marqué l'opinion publique ${ }^{39}$, le gouvernement a souhaité encourager le développement de ces groupes bénévoles, via un projet intitulé «Plan accéléré pour la sécurité et la tranquillité des enfants» (Kodomo anzen anshin kasokuka puran 子ども安全·安心加速化プラン). Ceci montre qu'aux yeux des acteurs publics, le quartier est appelé à jouer un rôle décisif dans les domaines suivants : protéger les enfants contre la délinquance, développer leur "indépendance» (shutaisei 主体性 ${ }^{40}$ ), leur «sociabilité» (shakaisei 社会性) et leur «état d'esprit» (kenzen na kokoro 健全な心). Impliqués dans ce projet, le ministère de l'Éducation, de la Culture, des Sports, des Sciences et de la Technologie (MEXT Monbu kagakushō 文部科学省) et le ministère de la Santé, du Travail et des Affaires sociales (MHLW Kōsei rōdoshō 厚生 労働省) ont ainsi voulu favoriser une plus grande participation des parents et plus particulièrement des mères de famille. Le gouvernement a également enrichi le contenu du "carnet d'éducation familiale» (katei kyōiku techo $\overline{0}$ 家庭教育手帳) distribué à tous les parents dont les enfants n'ont pas encore l'âge d'aller au lycée. Le carnet précise ainsi que "pour se protéger, il importe de connaître le danger» et que «la famille doit enseigner aux

38. Ce système de prix se fonde sur les recommandations de chaque commissariat local, ce qui renforce les liens entre les niveaux local et national.

39. En 2004 et 2005, trois assassinats ont attiré l'attention du public : dans les trois cas, il s'agissait de l'assassinat d'une fille de 7 ans tuée sur le chemin du retour de l'école (dans les départements de Hiroshima, de Tochigi et de Nara). La communication de la police a mis l'accent sur la pédophilie pour ces trois meurtriers.

40. Le terme "shutaisei 主体性» est utilisé dans l'idée qu'il est important que les adolescents développent leur autonomie (jiritsusei 自立性) tout en apprenant les règles de vie en commun permettant, d'après le programme, de mieux connaître la société réelle (jisshakai 実社会). 
enfants les moyens de se protéger contre les dangers et les accidents ${ }^{41}$ ». Cette attention portée à la sécurité des enfants a entraîné, dans l'ensemble, une forte mobilisation. Cela peut se lire au premier chef dans la proportion croissante de groupes de parents d'élèves. D'après des enquêtes réalisées en 2004, 53,1 \% des 19515 groupes interrogés étaient des chōnaikai, 14,2 \% des groupes de parents d'élèves, et 15,1\% correspondaient à d'autres groupes. Mais, un an plus tard, en 2005, le nombre de groupes de parents d'élèves était multiplié par 4,2 2 .

\section{Les pratiques bénévoles développées dans le cadre d'actions dites préventives}

L'augmentation du nombre de groupes repose ainsi sur un contexte préparé au niveau national, d'abord par la police puis par le gouvernement. Cependant, afin de mieux examiner le développement des actions préventives, il est important d'observer la manière dont les habitants ont accepté de se mobiliser pour prendre en charge une partie de la prévention de la délinquance.

Le plus souvent, les habitants réalisent des patrouilles au sein du quartier en échangeant des salutations. D'après les participants, ces salutations visent d'une part à augmenter le contrôle de l'environnement immédiat et d'autre part à éduquer les enfants pour qu'ils apprennent à maîtriser les échanges interpersonnels. L'hypothèse commune à ces formes d'action n'est donc pas d'arrêter directement des délinquants mais bien plutôt de les dissuader de passer à l'acte.

À partir de l'analyse des dossiers de trois cent cinquante-deux groupes d'habitants, nous avons pu classer ces derniers en trois types distincts selon le profil de leurs membres : le premier type correspond à des actions gérées

41. Mesures pour protéger les enfants contre le crime (Hanzai kara kodomo o mamoru tame no taisaku 犯罪から子供を守るための対策) : réunion de coordination interministérielle, 20 décembre 2005.

42. Dossier publié par l'Agence nationale de la police, concernant les activités des habitants du quartier et les groupes bénévoles réalisant des actions préventives par euxmêmes, 31 décembre 2005. 
par les chōnaikai, le deuxième à des actions gérées par les organisations locales non traditionnelles, et enfin le troisième aux activités menées par des associations de commerçants. À partir de cette tripartition d'ensemble, des entretiens semi-directifs ont été réalisés auprès d'une cinquantaine de bénévoles participant aux actions. Plus spécifiquement, des observations directes ont été menées au sein de treize groupes actifs dans une zone périurbaine de Tokyo, d'un groupe de la ville de Sakai à Osaka ainsi que d'un groupe à Kōbe $^{43}$. Les quartiers résidentiels observés ont pour caractéristique de faire partie de quartiers considérés comme favorisés et/ou agréables à vivre.

\section{Une intégration différenciée entre organisations traditionnelles ${ }^{44}$ et non-traditionnelles}

Les entretiens semi-directifs nous ont d'abord permis de comprendre que les bénévoles ont entrepris ces actions à cause d'un décalage entre deux images de leur quartier : celle qu'ils en ont en tant qu'habitants et celle qui se trouve, à un moment donné, diffusée publiquement. La prise de conscience d'un tel décalage est en grande partie liée soit à la diffusion d'informations sur le taux de criminalité en fonction des quartiers, notamment à partir des années 2000, soit à des échanges avec des voisins évoquant la dégradation de la sécurité de leur quartier. Dans tous les cas étudiés, c'est cette diffusion d'informations qui a favorisé le passage d'une préoccupation individuelle à une question relevant de la communauté de quartier et donc

43. Nous avons ainsi réalisé des enquêtes de terrain auprès de quinze groupes. Ils se distinguent de la façon suivante : neuf groupes s'inscrivent dans le premier type (huit groupes à Tokyo et un à Kōbe, créés de 1997 à 2005); deux groupes correspondent au deuxième type (un groupe dans la ville basse de Tokyo et un autre dans la ville de Sakai à Osaka, créés respectivement en 2002 et 2003); enfin, quatre groupes répondent au troisième type (actifs autour des gares de Shibuya, Meidaimae, Machida et Tokiwadai de Tokyo, ils ont été créés de 2002 à 2006). Le choix de ces groupes s'est fait dans l'objectif de pouvoir observer l'influence de certains groupes précurseurs sur la formation d'autres groupes.

44. Dans cet article, l'expression "organisations traditionnelles» désigne notamment les shōtengai shinkō kumiai 商店街振興組合 (Syndicat pour le développement des rues commerçantes), les chōnaikai 町内会, communautés de quartier, autrement appelées jichikai 自治会. 
appelant une prise en charge collective. On peut ainsi prendre l'exemple d'une personne victime d'un cambriolage mais qui n'osait en faire part à ses voisins, et se reprochait de n'avoir pas suffisamment fait preuve de prudence $^{45}$. Cependant, la diffusion publique d'informations à ce sujet lui a permis de prendre conscience que sa situation ne résultait pas uniquement de son imprudence, mais relevait d'un problème plus large.

Dans ces cas, c'est un choc lié au sentiment d'insécurité qui conduit ces bénévoles à agir. On remarque en outre un fort sentiment d'appartenance au quartier, car tous les chefs d'équipe interrogés, qui sont particulièrement impliqués, vivent depuis plus de vingt ans sur les mêmes lieux et ont noué à cette occasion de nombreux liens de voisinage. Ils ont pu ainsi s'impliquer dans les activités au sein d'un chōnaikai, dans leurs responsabilités de parents d'élèves, ou dans l'animation des commerces.

Nous pouvons cependant constater des développements différenciés en fonction des types d'organisations : en zone résidentielle, lorsqu'il s'agit de personnes ne relevant pas des structures traditionnelles considérées comme légitimes, elles peuvent tout d'abord être confrontées à un conflit de légitimité. Citons, à cet égard, le propos d'une responsable de groupe :

Tout au début, ceetait vraiment difficile. On nous disait entre autres : "Mais qu'est-ce que vous faites?", "Rentrez à la maison!"... J'y suis allée avec un agent de police mais le chef du chönaikai n'était pas convaincu. Pour faire quelque chose de nouveau, c'était vraiment difficile... Mais, maintenant, c'est de mieux en mieux reconnu. (Entretien semi-directif avec la responsable d'équipe d'un groupe de prévention à Tokyo.)

Nous constatons ici que le groupe a eu besoin de l'appui de la police pour légitimer son action auprès des habitants. De son côté, la police éprouvait elle aussi le besoin de se rapprocher des habitants suite à une baisse de confiance à son égard, ce que révèle cet extrait d'entretien avec un policier de la ville de Sakai. Le propos est centré sur l'importance de renouer des liens de confiance avec les habitants, de restaurer une proximité perdue :

45. Entretien semi-directif avec la responsable d'équipe d'un groupe de prévention à Tokyo. 
Avant, le simple fait de faire partie de la police inspirait confiance aux gens. Maintenant, ce n'est plus le cas. Nous ne pouvons pas avancer seuls. [...] Et surtout, pour gagner leur confiance, il faut toujours adopter le point de vue des habitants. La présence du bénévolat nous motive beaucoup, du coup. Nous avons donc accepté d'avoir quelqu'un qui nous fait part des mécontentements. [...] Nous avons ressenti le danger qu'il y aurait à ne plus être respectés pour notre travail. (Entretien semi-directif avec un policier d'un commissariat de la ville de Sakai).

On peut par ailleurs ajouter que la progression des structures non-traditionnelles n'a pas été sans conséquences sur l'organisation de certains chōnaikai qui n'assuraient jusqu'alors que peu d'activités liées à la sécurité. Lorsque les nouveaux groupes ont commencé à être reconnus au sein du quartier, certains chōnaikai ont été conduits à infléchir leurs activités dans une direction plus sécuritaire. Ceci a pu ici et là aboutir à un rapprochement des deux types de structures sans que disparaisse pour autant la hiérarchie entre organisations traditionnelles et non-traditionnelles :

Il y a aussi une section de prévention de la délinquance dans le chōnaikai. Mais cétait juste un nom et elle ne faisait rien. Tout d'un coup, le chef du chōnaikai est venu chez moi pour me demander si les membres pouvaient porter la même veste ${ }^{46}$. J'ai été très surprise. [...] Mais si je me positionne de manière supérieure par rapport au chōnaikai, ou que je suis hautaine, ils vont dire : "Mais qu'est-ce que c'est que ce groupe ?". Je reste donc toujours à ma place. (Entretien semi-directif avec la responsable d'équipe d'un groupe de prévention à Tokyo.)

La mobilisation des habitants va de pair avec le mouvement initié par les policiers, mais ceci a été également un moyen, pour les groupes non-traditionnels, de se faire reconnaître comme étant les acteurs principaux de la sécurité du quartier. Il faut en revanche préciser que même l'appui des policiers ne pousse pas les habitants à s'inscrire dans une chaîne hiérarchique, par rapport à la police elle-même ou bien à une collectivité locale.

46. La plupart des acteurs bénévoles portent des vestes identiques afin de renforcer la visibilité de leurs actions. Cette veste est souvent fournie par la police ou par la municipalité. 


\section{L'implication des «anciens » du quartier : des enjeux à la fois territoriaux et personnels}

Les bénévoles défendent l'idée qu’ils sont autonomes, responsables de l'image de leur ville de même que de leurs propres enfants. Ils insistent régulièrement sur le fait que les pouvoirs publics n'ont pas connaissance de la réalité de leur quotidien. Ce faisant, ils tendent à se considérer comme les nouveaux acteurs de la société civile, véritables représentants des habitants, d'un point de vue plus large que celui des fonctionnaires considérés comme «novices» au sein du quartier. Ils développent alors des actions visant à ranimer une forme de fierté locale, fondée sur une mémoire partagée et qui se trouve redécouverte ou ravivée à chaque patrouille. À Tokyo, selon les quartiers, l'accent est mis sur des éléments différents : dans l'arrondissement de Suginami, l'importance est accordée aux espaces verts, familiers des riverains; tandis que, dans un quartier populaire de la shitamachi, il s'agit surtout d'échanges intergénérationnels à l'occasion de fêtes locales. À travers ces actions, ils aspirent à remodeler la ville en agissant sur l'environnement urbain.

Les « anciens» tendent quant à eux à se positionner en rivalité par rapport aux habitants d'un autre périmètre, ce qui est particulièrement visible chez les chefs des groupes. Leur mémoire du quartier entretient en outre un lien fort avec le sentiment de détenir une conscience pédagogique que les écoles et les familles d'aujourd'hui ne possèdent plus. Ces "anciens" accordent ainsi une importance spécifique à la formation. Ils s'adressent souvent aux enfants, notamment dans le cadre des salutations déjà décrites. Redoutant le vieillissement de la population locale, ils considèrent les enfants comme les "fruits» du quartier et cherchent à développer chez eux un sentiment de solidarité susceptible de les y ramener plus tard. Ils entendent également leur inculquer le respect de l'autre (omoiyari 思いやり) et l'indépendance (shutaisei 主体性). Cette démarche permet au demeurant de répondre à certains objectifs des établissements scolaires pour favoriser les échanges intergénérationnels dans la perspective du développement personnel de l'enfant.

Par ailleurs, pour les membres plus âgés en particulier, la mission initiale de réduction de la délinquance s'est rapidement doublée d'une autre mission; celle d'entretenir les liens avec les autres habitants, permettant de dissiper d'autres peurs, plus personnelles. Certains disent profiter de cette activité pour entretenir leur santé en marchant au moins une heure par 
semaine. D'autres y voient l'occasion de discuter avec les voisins afin de rompre le sentiment de solitude et d'éviter l'isolement en cas de désastre naturel. Certains travailleurs sociaux prétextent le maintien de la sécurité pour stimuler une personne âgée repliée sur elle-même : le ramassage de déchets ou les patrouilles constituent des activités relativement accessibles. Enfin, pour beaucoup d'hommes retraités, ces missions comblent le sentiment de perte de statut professionnel.

De plus, il arrive qu'au sein d'un même groupe se forment des sousgroupes par affinités, qui se réunissent pour discuter, voire partent ensemble en voyage. Les patrouilles permettent à l'occasion de développer les relations, d'échanger des informations et d'approfondir ses réseaux en fonction des orientations politiques, des passe-temps ou des difficultés de la vie quotidienne. Les habitants apprécient leur expérience au sein des groupes préventifs en les comparant avec les activités habituelles des chönaikai «dans lesquels il y a une hiérarchie comme dans une entreprise». Ils insistent sur le caractère bénévole de leurs groupes et sur la possibilité de se retirer lorsqu'ils le souhaitent. C'est là une autre différence avec les tâches habituelles des chōnaikai, notamment lorsque les participants considèrent que les actions préventives n'en dépendent pas $^{47}$. Ils voient ainsi dans les actions préventives l'expression d'une société civile marquée par davantage de liberté.

L'exemple des actions menées dans la ville de Sakai illustre bien cette conscience d'être des acteurs civils. Les patrouilles mensuelles impliquant environ cent participants : habitants, acteurs publics et policiers. Certains résidents profitent de ce temps de marche pour transmettre leurs doléances aux agents municipaux et aux policiers, tandis que d'autres discutent simplement avec leurs voisins.

Ainsi, les groupes de maintien de sécurité sur lesquels ont porté nos enquêtes ont souvent acquis, au cours de leur développement, une deuxième dimension au caractère plus social. Autrement dit, certains participants accordent beaucoup plus de valeur au renforcement des liens

47. Cette tendance au développement de la sociabilité est moins forte dans les groupes fortement liés au chōnaikai. Même si, initialement, ces programmes ont commencé en ne sélectionnant que les personnes les plus motivées, la majorité des participants considèrent aujourd'hui les actions préventives comme faisant partie intégrante de leurs activités de chōnaikai. 
de voisinage plutôt qu'aux effets des patrouilles sur la sécurité. La prévention représente à leurs yeux l'activité la plus facile parce qu'elle ne nécessite pas de technique spécifique et qu'elle leur permet de trouver une raison d'être au sein de la société locale. Nous pouvons ainsi constater une forme d'inversion des fins et des moyens entre discours public et pratique réelle : le slogan "machizukuri» avait pour objectif de faire baisser la criminalité par le renforcement des liens sociaux; dans la pratique, la prévention de la criminalité semble plutôt constituer un outil afin de tisser des liens sociaux au sein du quartier. Et cette dimension elle-même est indéniablement soutenue par les discours des acteurs publics, qui mettent en avant l'importance de la solidarité locale en matière de maintien de l'ordre. Toutefois, un tel dispositif sécuritaire présente des risques de dérive, en ce qu'il confere à une certaine fraction de la population locale le droit d'exercer la fonction d' «adultes légitimes», une légitimité auto-conférée qui pourrait être déniée ou contestée par d'autres membres de la communauté.

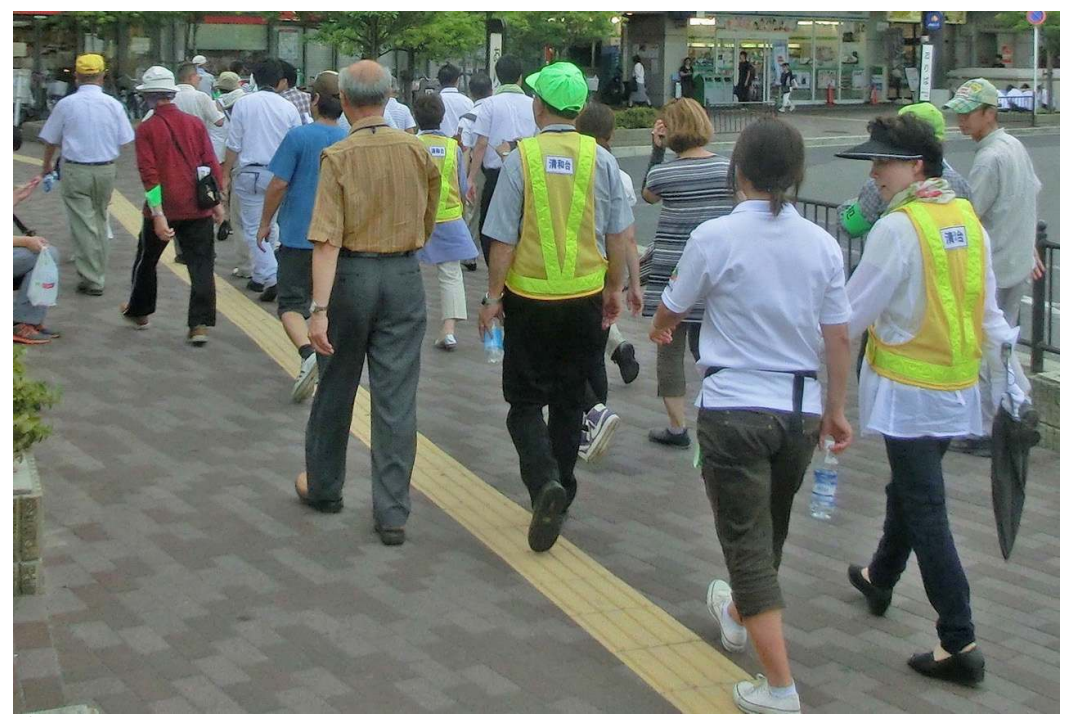

Fig. 2

Scène extraite des patrouilles mensuelles d'un groupe de prévention dans la ville de Sakai, auxquelles participent des habitants, des agents municipaux, des policiers, des enseignants des institutions scolaires, etc. 


\section{Conclusion}

À la différence de ce qu'il s'est passé pendant la période militariste, au cours de laquelle les communautés de quartier exerçaient un rôle de surveillance mutuelle, les chōnaikai ne se bornent plus à être de simples organisations placées au bas d'une hiérarchie dominée par les pouvoirs publics. Cependant, dans les zones urbaines, nous avons constaté un renforcement de la dimension sécuritaire des chōnaikai, et une continuité par rapport à la période ancienne. Tout en recherchant, dans les pays occidentaux, un modèle en matière de maintien de l'ordre, le dispositif japonais implique le domaine moral et la vie sociale de la population. Si l'attitude actuelle de la police et du gouvernement repose sur l'idée que la criminalité est réduite par cette «exception japonaise» qu'est la solidarité locale, il faut rappeler que le taux de délinquance était plus élevé à la période où cette solidarité était plus forte.

Cette politique de prévention a en partie pour but d'absorber les préoccupations sociales et de réduire les contestations. Ainsi, après le premier choc pétrolier, on a vu se développer la notion de "société du bienêtre» (Nihongata fukushi shakai 日本型福祉社会). Avec la fin de la Haute Croissance économique, le système de l'État providence s'est progressivement modifié, notamment en matière de retraite et de santé (Takegawa 2012). Cette question doit être reliée à la politique menée à l'égard des personnes âgées au milieu des années 80 , lesquelles ont été perçues à la fois comme victimes de la criminalité et acteurs potentiels de la prévention, à travers leurs activités bénévoles. Concernant les nouvelles générations, au lieu de chercher à construire des politiques sociales, la police a plutôt pointé la responsabilité des jeunes et de leur famille, en évoquant le déclin moral. Cela a justifié la participation de personnes âgées investies d'un rôle pédagogique. Par ailleurs, sur le plan urbanistique, les acteurs publics ont renforcé la dimension de sécurité civile en matière d'équipement et en termes de relations humaines. Le séisme de Hanshin-Awaji, à la suite duquel de nombreuses personnes se sont mobilisées, a permis aux autorités de présenter les membres des groupes de prévention comme de nouveaux acteurs civils.

Ces bénévoles, partageant la conscience d'être de nouveaux acteurs, cherchent alors à établir des liens dépourvus de contrainte, à la différence des tâches habituelles des organisations traditionnelles. Mais en observant 
leurs raisons d'agir, nous pouvons reprendre l'observation de Foucault (2004) suivant laquelle les individus sont amenés par le politique à agir comme s'ils agissaient de leur propre volonté, sans qu'il y ait besoin d'un recours effectif à des moyens contraignants (Foucault 2004 : 361) ou encore, celle de Dreyfus (1976 : 169) et de son dispositif disciplinaire "d'autant plus efficace qu'[il] est mieux intérioris[é] et moins visible», dans un espace urbanistique «rationnalisé et fonctionnaliste». Face à cette préoccupation sécuritaire, on pourrait ainsi s'interroger sur le signe d'un «glissement de l'État social vers l'État sécuritaire» (Castel 2003 : 56), ou à tout le moins d'un renforcement de l'État sécuritaire, guidé par la volonté de créer un environnement protecteur aux niveaux à la fois sécuritaire, social et sanitaire. Les actions préventives représenteraient un moyen de répondre à des inquiétudes, alors que celles-ci viennent, plus fondamentalement, d'un manque d'État social. Le pouvoir disciplinaire donné aux habitants fait ainsi «marcher un pouvoir relationnel qui se soutient lui-même par ses propres mécanismes» (Foucault 1975 : 179). La visée ultime d'un tel dispositif serait de responsabiliser habitants et organisations locales, sans que l'État ait besoin de se confronter aux questions sociales. 


\section{Bibliographie}

\section{AJISAKA Manabu 鯵坂学 2006}

«Chiiki jūmin soshiki to chiiki gabanansu» 地域住民組織と地域ガバ ナンス (Les organisations locales des habitants et la gouvernance locale), in Yazawa Sumiko 矢澤澄子 \& Iwazaki Nobuhiko 岩崎信彦 (dir.), Chiiki shakai no seisaku to gabanansu 地域社会の政 策とガバナンス (Les politiques dans la société locale et sa gouvernance), Tokyo, Tōshindō 東信堂 : 173-187.

\section{CARRÉ Guillaume 2003}

"Fêtes et logiques territoriales dans les quartiers d'Edo au xIx ${ }^{\mathrm{e}}$ siècle », Annales. Histoire, Sciences Sociales, 58 (5) : 1057-1078.

\section{CASTEL Robert 2003}

L'insécurité sociale : qu'est-ce qu'être protégé?, Paris, Le Seuil, 96 p.

\section{DREYFUS Jacques 1976}

La ville disciplinaire, Paris, Galilée, 215 p.

\section{FOUCAULT Michel 1975}

Surveiller et punir, Paris, Gallimard, 352 p.

\section{FOUCAULT Michel 2004}

Sécurité, territoire, population, Paris, Le Seuil, 448 p.

IWASAKI Nobuhiko 岩崎信彦 et al. 1989 Chōnaikai no kenkyū 町内会の研究 (Recherches sur les chōnaikai), Tokyo, Ochanomizu shobō 御茶の水書房, 484 p.
HAMAI Kōichi 浜井浩一 \& SERIZAWA Kazuya 芹沢一也 2006 Hanzai buan shakai 犯罪不安社会? (La société du sentiment d'insécurité), Tokyo, Kōbunsha 光文社, 249 p.

\section{HAMAI Kōichi 浜井浩一 2013}

Naze hanzai wa genshō shiteirunoka な ぜ犯罪は減少しているのか (Pourquoi la criminalité est-elle en baisse?), Tokyo, Hanzai shakaigaku kenkyū 犯罪社会学研 究, 38:53-77.

\section{HISHIYAMA Kōsuke 菱山宏輔 2014} «1960 nendai zenhan ni okeru Tōkyō-to chōnaikai no jichiishiki to sono hōsetsu » 1960年代前半における東京都町内会の 自治意識とその包摂 (La conscience de l'autonomie et la mobilisation des organisations de voisinage à Tokyo dans la première moitié des années 1960), Chiiki shakaigakkai nenpō地域社会学会 年報, 26:91-104.

\section{KAWAI Mikio 河合幹雄 2004} Anzen shinwa hōkai no paradokkusu 安全 神話崩壊のパラドックス (Le paradoxe de la destruction du mythe de la sécurité), Tokyo, Iwanami shoten 岩波書店, 320 p.

\section{MAILLARD Jacques (de) \& LE GOFF} Tanguy 2009

"La tolérance zéro en France ", Revue française de science politique, 59 : 655-679.

\section{NAKATA Minoru 中田実 2000}

Sekai no jūmin soshiki 世界の住民組 織 (Les communautés locales dans le monde), Tokyo, Jichitai kenkyū sha 自治 体研究社, 287 p. 


\section{OBINATA Sumio 大日方純夫 1993}

Keisatsu no shakaishi 警察の社会史 (Histoire sociale de la police), Tokyo, I wanami shoten 岩波書店, $230 \mathrm{p}$.

\section{ROCHÉ Sébastian 2000}

"La théorie de la "vitre cassée" en

France», Revue française de science politique, 50 : 387-412.

SHIMIZU Masahiko 清水雅彦 2007 Chian seisaku to shite no " anzen anshin machi-zukuri»-kanshi to kanri no shōhai 治安政策としての「安全·安心まちづくり」一 監視と管理の招牌 (《La fabrique d'une ville sûre et paisible » dans le cadre de la politique de sécurité : le tableau de surveillance et de gestion), Tokyo, Shakai hyōronsha, 社会評論社, 333 p.

TAKEGAWA Shōgo 武川正吾 2012 Fukushi shakaigaku no sōzōryoku 福祉社 会学の想像力 (L'imagination des études sociologiques sur l'action sociale), Tokyo, Kōbundō 弘文堂, 160 p.

TSUJINAKA Yutaka 辻中豊 et al. 2009 Gendai nihon no jichikai chōnaikai 現代 日本の自治会·町内会 (Les jichikai et les chōnaikai dans le Japon contemporain), Tokyo, Bokutakusha 木鐸社, 259 p.

YOSHIHARA Naoki 吉原直樹 2011 Komyuniti sutadizu コミュニティスタ ディーズ (Community studies), Tokyo, Sakuhinsha 作品社, 391 p.

WATANABE Osamu 渡辺治 2005 "Gurōbaruka. "Tsuyoi kokka" seisaku to gendai keisatsu no nerai »
グローバル化・強い国家」政策と現代警 察のねらい (Globalisation, politiques de l'«État fort» et enjeux policiers d'aujourd'hui), in Ogura Toshimaru 小倉 利丸 (dir.), Gurōbaruka to kanshi keisatsu kokka e no teikō グローバル化と監視警察 国家への抵抗 (Globalisation et résistance contre l'État de surveillance policière), Tokyo, Kinohanasha 樹花舎 : 13-118. 
